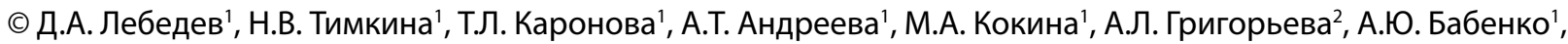
Е.Н. Гринева

1Национальный медицинский исследовательский центр им. А.А. Алмазова, Институт эндокринологии, Санкт-Петербург, Россия

${ }^{2}$ Клиническая ревматологическая больница №25, Санкт-Петербург, Россия.

Обоснование. Ингибиторы натрий-глюкозного ко-транспортера 2 типа (ИНГТ2) являются сахароснижающими препаратами и зарегистрированы для лечения сахарного диабета 2 типа (СД2). В ходе клинических исследований данных препаратов были получены доказательства повышенного риска переломов и влияния на уровни фосфора, витамина D и паратиреоидного гормона (ПТГ).

Цель. В связи с этим целью данного исследования было изучить влияние наиболее селективного ИНГТ2 эмпаглифлозина на параметры фосфорно-кальциевого обмена у пациентов с СД2 и сохранной функцией почек.

Материалы и методы. Тридцать девять пациентов с СД2 получали эмпаглифлозин в дозе 10 мг в дополнение к сахароснижающим препаратам в течение 12 нед. Перед началом лечения была проведена двухэнергетическая рентгеновская абсорбциометрия (DXA) с оценкой трабекулярного костного индекса (ТKИ). В начале и в конце исследования оценивали концентрацию фосфора (Р), общего (оСа) и ионизированного кальция (Са $\left.{ }^{++}\right)$, фактора роста фибробластов-23 (ФРФ-23), 25(ОН)D и ПTГ.

Результаты. По результатам DXА только у 2 пациентов имел место остеопороз, у 10 (25,6\%) пациентов показатели минеральной плотности кости (МПК) были ниже 1,35 г/см² по шкале ТКИ. На фоне лечения эмпаглифлозином в течение 12 нед было получено значимое увеличение ФРФ-23. По сравнению с исходным уровнем не было получено статистически значимых различий в концентрациях $\mathrm{P}$, оCa, $\mathrm{Ca}^{++}$, ПТГ и 25(OH)D через 12 нед лечения. Уровень ФРФ-23 не коррелировал с уровнем скорости клубочковой фильтрации ни до, ни после лечения ( $r=0,31, p=0,27$ и $r=0,39, p=0,55$ соответственно). Кроме того, исходный уровень МПК с поправкой на ТКИ и исходный уровень 25(OH)D не коррелировали с концентрациями Са, Р, ФРФ-23 и ПтГ ( $>>0,05)$.

Заключение. Таким образом, результаты исследования продемонстрировали влияние эмпаглифлозина на увеличение уровня ФРФ-23 без значительного изменения концентрации фосфора, кальция, 25(OH)D и ПТГ после 12 нед лечения у пациентов с СД2 и сохраненной функцией почек. Полученные данные подтвердили необходимость оценки ТКИ у больных СД2 для получения дополнительной информации о качестве костной ткани.

КЛЮЧЕВЫЕ СЛОВА: сахарный диабет 2 типа; эмпаглифлозин; кальций; фактор роста фибробластов-23.

\title{
EFFECT OF EMPAGLIFLOZIN ON PHOSPHORUS AND CALCIUM METABOLISM IN PATIENTS WITH TYPE 2 DIABETES MELLITUS WITH PRESERVED KIDNEY FUNCTION
}

(c) Denis A. Lebedev'1, Natalia V. Timkina'1, Tatiana L. Karonova', Alena T. Andreeva', Maria A. Kokina', Alexandra L. Grigorieva², Alina Yu. Babenko', Elena N. Grineva'

${ }^{1}$ Almazov National Medical Research Centre, Institute of Endocrinology, St. Petersburg, Russia

${ }^{2}$ Clinical Rheumatological Hospital № 25, St. Petersburg, Russia

Background: Sodium glucose co-transporter type 2 inhibitors (iSGLT2) are antihyperglycemic drugs approved for the treatment of type 2 diabetes mellitus (T2DM). Clinical trials with these drugs have shown evidence of an increased risk of fractures and an effect on phosphorus, vitamin D and parathyroid hormone (PTH) levels.

Aim: The aim of this study was to investigate the effect of the most selective iSGLT2 empagliflozin on the calcium and phosphorus metabolism in patients with T2DM and preserved kidney function.

Materials and methods: Thirty-nine T2DM patients were received empagliflozin $10 \mathrm{mg}$ in addition to their antihyperglycemic drugs for 12 weeks. Before starting treatment, a dual-energy X-ray absorptiometry (DXA) with an assessment of the trabecular bone score (TBS) was performed. The concentration of phosphorus (P), total (tCa) and ionized calcium (Ca ${ }^{++}$), fibroblast growth factor 23 (FGF-23), 25(OH)D and PTH were assessed.

Results: According to the DXA results, only 2 patients had osteoporosis, 10 (25.6\%) patients had bone mineral density (BMD) values below $1.35 \mathrm{~g} / \mathrm{cm}^{2}$ on the $\mathrm{TCl}$ scale. Treatment with empagliflozin for 12 weeks was lead to significant increase in FGF-23. Compared to the baseline level, there were no statistically significant differences in the concentrations of $\mathrm{P}$, oCa, $\mathrm{Ca}^{++}$, PTH and 25(OH)D after 12 weeks of treatment. The level of FGF-23 did not correlate with the level of glomerular filtration rate either before or after treatment $(r=0.31, p=0.27$ and $r=0.39, p=0.55$, respectively). In addition, baseline BMD adjusted for TBS and baseline 25(OH)D did not correlate with Ca, F, FGF-23, and PTH concentrations ( $p>0.05)$. 
Conclusion: Thus, empagliflozin has increased the level of FGF-23 without significant changes in the concentration of phosphorus, calcium, $25(\mathrm{OH}) \mathrm{D}$, and PTH after 12 weeks of treatment in patients with T2DM and preserved renal function. The obtained data confirmed the necessity to assess the TBS in patients with T2DM, because it's provide additional information on the quality of bone tissue.

KEYWORDS: type 2 diabetes mellitus; empagliflozin; calcium; fibroblast growth factor-23.

\section{ОБОСНОВАНИЕ}

Известно, что пациенты с сахарным диабетом 2 типа (СД2) имеют повышенный риск переломов в связи со множеством причин, таких как гипергликемия, хронические микрососудистые осложнения, дефицит витамина D, повышенный риск падений и использование некоторых сахароснижающих препаратов (тиазолидиндионы) [1-2]. Кроме того, инсулинорезистентность, хроническое вялотекущее воспаление и образование конечных продуктов гликирования оказывают значительное влияние на изменения структуры костей и последующий риск переломов [3]. Данные различных исследований свидетельствуют о том, что СД2 увеличивает риск переломов при более высоких значениях минеральной плотности костной ткани (МПК), нежели в общей популяции [4-5]. Однако оценка МПК не может полностью продемонстрировать изменения, происходящие в трабекулярной части костной ткани, в связи с чем был предложен новый способ оценки состояния кости - трабекулярный костный индекс (ТКИ) [6]. В некоторых исследованиях сообщалось о снижении показателя ТКИ у пациентов с СД2 даже при нормальных показателях МПК [7-9].

В последние годы у больных СД2 все большее внимание уделяется влиянию антигипергликемических препаратов на состояние костей. В октябре 2015 г. Управление по санитарному надзору за качеством пищевых продуктов и медикаментов США (FDA) выпустило предупреждение о возможном риске переломов, связанных с приемом канаглифлозина [10]. Ингибиторы натрий-глюкозного ко-транспортера 2 типа (ИНГТ2) являются широко используемыми сахароснижающими препаратами, которые ингибируют реабсорбцию глюкозы в проксимальных извитых канальцах нефронов и снижают уровень глюкозы в крови инсулиннезависимым путем [11]. Данные по улучшению сердечно-сосудистых исходов и замедлению прогрессирования хронической болезни почек на фоне терапии ИНГТ2 позволяют поставить данную группу препаратов на первое место при выборе сахароснижающей терапии [12-14]. Несколько ИНГТ2, включая канаглифлозин, дапаглифлозин и эмпаглифлозин, доступны во многих странах, включая Российскую Федерацию.

Результаты проведенных клинических исследований показали, что, несмотря на наличие плейотропных эффектов в отношении сердечно-сосудистой системы и почек, на фоне терапии ИНГТ2 возможно повышение уровня фосфора (Р) в сыворотке крови, а использование канаглифлозина может быть ассоциировано даже с повышением риска переломов [15-17]. Дополнительно к этому результаты рандомизированного перекрестного исследования у здоровых людей продемонстрировали, что канаглифлозин оказывает влияние на уровень фактора роста фибробластов-23 (ФРФ-23), 1,25-дигидроксивитамина D $\left(1,25(\mathrm{OH})_{2} \mathrm{D}\right)$ и паратиреоидного гормона (ПТГ) [18].
Стоит отметить, что канаглифлозин влияет не только на натрий-глюкозные ко-транспортеры 2-го типа (НГТ2), но и на рецепторы 1-го типа тоже. Тем не менее в последнем исследовании канаглифлозина CREDENCE не было получено данных об увеличении числа переломов костей при приеме канаглифлозина по сравнению с плацебо [19].

\section{ЦЕЛЬ ИССЛЕДОВАНИЯ}

Целью настоящего исследования было изучить влияние наиболее селективного и широко используемого ИНГТ2, эмпаглифлозина, на параметры фосфорно-кальциевого обмена у больных СД2 с сохранной функцией почек.

\section{МАТЕРИАЛЫ И МЕТОДЫ}

\section{Место и время проведения исследования}

Место проведения. Исследование проводилось на базе НМИЦ им. В.А. Алмазова, проведение денситометрии осуществлялось на базе Ревматологической больницы №25.

Время исследования. Исследование проводилось в 2018-2019 гг.

Критерии включения: женщины или мужчины в возрасте от 45 до 65 лет; диагноз СД2 установлен не менее 1 года назад; уровень гликированного гемоглобина $\left(\mathrm{HbA}_{1 c}\right)$ 7,5-10,0\%; стабильная сахароснижающая терапия в течение 12 нед до включения в исследование.

Критерии исключения: острые заболевания или обострение хронических заболеваний на момент скрининга; анамнез рецидивирующих инфекций мочевыводящих путей; сердечно-сосудистое событие в течение последних 6 мес; хроническая болезнь почек (расчетная скорость клубочковой фильтрации (рСКФ) по данным CKD-EPI <60 мл/мин/1,73 м²); первичный гиперпаратиреоз; прием диуретиков; терапия пиоглитазоном или инсулинотерапия; ревматологические заболевания и прием глюкокортикостероидов.

\section{Дизайн исследования}

Исследование было одноцентровым, с проспективным наблюдением пациентов.

\section{Методы}

Перед началом лечения для всех пациентов был рассчитан 10-летний риск перелома шейки бедра и основных остеопоротических переломов при помощи шкалы FRAX. При расчете риска по шкале FRAX использовалась МПК шейки бедра без учета ТКИ. В связи с наличием у пациентов СД использовалась поправка в виде ревматоидного артрита. Оценка риска проводилась согласно Федеральным клиническим рекомендациям по диагностике, лечению и профилактике остеопороза [20]. Также была 
проведена двухэнергетическая рентгеновская абсорбциометрия (DXA), включая измерение ТКИ. Лабораторно оценивались уровни $\mathrm{HbA}_{1 c}$ (Bio-Rad, США), креатинина, P, общего (оСa) и ионизированного кальция $\left(\mathrm{Ca}^{++}\right)$(Architect c4000 (Abbott, США)), ФРФ-23 (набор для ELISA FGF-23 (С-конец) BI-20702), 25-гидроксивитамина D (25(OH)D) (Abbott Architect 8000) и ПТГ (Biomerica, США). Образцы крови забирались утром натощак в начале и в конце 12-недельного периода лечения.

\section{Статистический анализ}

Статистический анализ проводили с использованием пакета статистических программ STATISTICA 10 (StatSoft Inc., США). Количественные данные представлены в виде медианы и интерквартильного размаха (Ме, 25\%; 75\% квартили), категориальные данные представлены частотами и процентами от общего числа наблюдений $\mathrm{n}$ (\%).
Сравнение исходных данных и данных последующего наблюдения проводилось с использованием критерия Уилкоксона. Корреляционный анализ проводился с использованием коэффициента ранговой корреляции Спирмена. Нулевая гипотеза отвергалась при $\mathrm{p}<0,05$.

\section{Этическая экспертиза}

Исследование было одобрено Этическим комитетом Национального медицинского исследовательского центра им. А.А. Алмазова (№020419 от 09 апреля 2018 г.).

\section{РЕЗУЛЬТАТЫ}

Исходные характеристики 39 пациентов и динамика показателей фосфорно-кальциевого обмена представлены в таблицах 1 и 2. Медиана $\mathrm{HbA}_{1 с}$ составила $8,9 \%$ $(8,1 ; 9,4)$, ИМТ - 29,1 кг/м² $(27,5 ; 34,9)$. Большинство

Таблица 1. Исходная характеристика пациентов

\begin{tabular}{|c|c|}
\hline Показатель & Значение, Ме [25; 75] \\
\hline Возраст, лет & $57,5[47,4 ; 62,8]$ \\
\hline Женский пол, n (\%) & $23(58,9)$ \\
\hline Мужской пол, n (\%) & $16(41,1)$ \\
\hline Длительность СД2, лет & $8,7[4,3 ; 10,6]$ \\
\hline 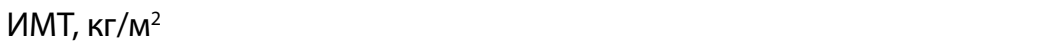 & $29,1[27,5 ; 34,9]$ \\
\hline Избыточная масса тела или ожирение, n (\%) & $31(76,9)$ \\
\hline СКФ, мл/мин/1,73 м² & $91,4[74,3 ; 106,1]$ \\
\hline $\mathrm{HbA}_{1 \mathrm{c}^{\prime}} \%$ & $8,9[8,1 ; 9,4]$ \\
\hline T-критерий $\left(\mathrm{L}_{1}-\mathrm{L}_{\mathrm{IV}}\right), \mathrm{SD}$ & $-0,8[-0,2 ;-1,7]$ \\
\hline$M \Pi K, \mathrm{~L}_{\mathrm{l}}-\mathrm{L}_{\mathrm{IV}}, \Gamma / \mathrm{CM}^{2}$ & $1,26[1,04 ; 1,38]$ \\
\hline Т-критерий (шейка бедра), SD & $-0,6[-0,1 ;-1,5]$ \\
\hline МПК, шейка бедра, г/см² & $0,98[0,86 ; 1,06]$ \\
\hline 10-летняя вероятность перелома проксимального отдела бедра, \% & $7,9 \%[4,8 ; 10,3]$ \\
\hline 10-летняя вероятность основных остеопоротических переломов, \% & $0,7 \%[0,3 ; 1,5]$ \\
\hline
\end{tabular}

Примечание. СД2 - сахарный диабет тип 2; ИМТ - индекс массы тела; СКФ - скорость клубочковой фильтрации; НbА рованный гемоглобин; $\mathrm{L}_{\mathrm{I}}-\mathrm{L}_{\mathrm{IV}}$ - поясничные позвонки I-IV.

Таблица 2. Показатели фосфорно-кальциевого обмена у больных сахарным диабетом 2 типа до и через 12 недель лечения эмпаглифлозином

\begin{tabular}{|c|c|c|c|}
\hline Показатели & $\begin{array}{l}\text { Исходно } \\
\text { Ме }[25 ; 75]\end{array}$ & $\begin{array}{c}\text { Через } 12 \text { недель лечения } \\
\text { Me }[25 ; 75]\end{array}$ & р-значение \\
\hline Фосфор сыворотки, ммоль/л & $1,08[0,85 ; 1,26]$ & $1,13[0,91 ; 1,29]$ & 0,059 \\
\hline Общий кальций, ммоль/л & $2,31[2,22 ; 2,49]$ & $2,34[2,26 ; 2,46]$ & 0,51 \\
\hline Ионизированный кальций, ммоль/л & $1,19[1,06 ; 1,30]$ & $1,22[1,11 ; 1,28]$ & 0,73 \\
\hline Паратгормон, пг/мл & $41,2[35,3 ; 61,2]$ & $44,1[33,5 ; 62,2]$ & 0,41 \\
\hline ФРФ-23, пмоль/л & $1,85[1,15 ; 2,64]$ & $2,21[1,37 ; 2,87]$ & 0.015 \\
\hline 25(OH)D, нг/мл & $19,9[12,8 ; 26,8]$ & $20,7[14,4 ; 27,1]$ & 0,68 \\
\hline \multicolumn{4}{|l|}{ Обеспеченность витамином D } \\
\hline Норма, n (\%) & $5(12,8)$ & $6(15,4)$ & \multirow{3}{*}{0,35} \\
\hline Недостаток, n (\%) & $25(64,1)$ & $26(66,6)$ & \\
\hline Дефицит, n (\%) & $9(23,1)$ & $7(18)$ & \\
\hline
\end{tabular}

Примечание. ФРФ-23 - фактор роста фибробластов-23; 25(ОН)D - 25-гидроксивитамин D. 
пациентов имели длительность диабета более 4 лет и получали комбинированную сахароснижающую терапию - метформин и препараты группы сульфонилмочевины или ингибиторы дипептидилпептидазы-4 (иДПП-4). Рассчитанная по шкале FRAX 10-летняя вероятность перелома шейки бедра и 10-летняя вероятность основных остеопоротических переломов составили $0,7 \%(0,3 ; 1,5)$ и $7,9 \%(4,8 ; 10,3)$ соответственно, что свидетельствовало о низком риске переломов у больных СД2. По результатам DXA, проведенной у всех больных, только у 2 пациентов диагностирован остеопороз,

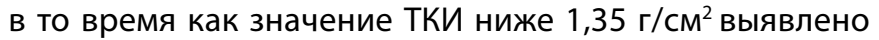
у 10 пациентов.

Через 12 нед лечения эмпаглифлозином наблюдалось значимое снижение $\mathrm{HbA}_{1 c}$ по сравнению с исходным уровнем - 8,1 $(7,6 ; 8,5)$ и 8,9 $(8,1 ; 9,4) \%, p=0,005$, а также снижение ИМТ - 27,1 $(24,9 ; 29,6)$ и $29,1(27,5 ; 34,9) \mathrm{kг} / \mathrm{M}^{2}$, $\mathrm{p}=0,0019$. Показатель СКФ значимо не изменился за время лечения и составил соответственно $91,4(74,3 ; 106,1)$ и 93,2 $(76,3 ; 104,2)$ мл/мин/1,73/м² $(p=0,4)$. Медиана показателя $\mathrm{Ca}^{++}$в сыворотке крови в начале лечения составила 1,19 $(1,06 ; 1,32)$ ммоль/л, Р в сыворотке крови - 1,02 (0,78; $1,26)$ ммоль/л. Уровень ПТГ был в пределах референсных значений у всех пациентов, хотя 74,4\% из них имели недостаточность или дефицит витамина D (см. табл. 2).

Наблюдалось незначительное увеличение уровня $\mathrm{P}$ крови через 12 нед лечения по сравнению с исходными данными, однако изменения не достигли статистической значимости. Вместе с тем на фоне лечения отмечалось значимое увеличение уровня ФРФ-23. По сравнению с исходным уровнем не было получено статистически значимой динамики концентраций $\mathrm{Ca}^{++}$, оCa, ПТГ и 25(OH)D через 12 нед лечения.

Корреляционный анализ не выявил связи между ФРФ-23 и уровнем СКФ ни до, ни после лечения ( $r=0,31$, $\mathrm{p}=0,27$ и $\mathrm{r}=0,39, \mathrm{p}=0,55$ соответственно). Также не выявлено корреляций между показателем МПК с поправкой на ТКИ и исходным уровнем 25(OH)D с такими показателями, как общий кальций, Р, ФРФ-23 и ПТГ ( $p>0,05)$.

\section{ОБСУЖДЕНИЕ}

\section{Репрезентативность выборок}

В исследование были включены пациенты с СД2, с сохранной функцией почек, получающие комбинированную терапию наиболее часто встречающимися таблетированными сахароснижающими препаратами. Несмотря на то что данная группа пациентов составляет большой процент среди всех пациентов с СД2, в исследовании не оценивались показатели фосфорно-кальциевого обмена у пациентов на монотерапии метформином и у пациентов, получающих инсулинотерапию.

\section{Сопоставление с другими публикациями}

Известно, что ИНГТ2, блокируя НГТ2 в проксимальных канальцах почек, способствуют увеличению экскреции глюкозы с мочой и снижению уровня глюкозы в крови [11]. В настоящее время отсутствуют данные по экспрессии НГТ2 в остеобластах или остеокластах. Взятые вместе, эти данные указывают на то, что ингибирование НГТ2 вряд ли будет играть важную функциональную роль в костной ткани. Считается, что ИНГТ2 в силу своих особенностей способны увеличивать транспорт фосфора через натрий-фосфатные ко-транспортеры в проксимальных канальцах почек [21]. Увеличение концентрации Р крови приводит к компенсаторному повышению уровня ФРФ-23, синтезирующегося в остеоцитах и являющегося, как известно, основным регулятором данного электролита, и в конечном итоге - к фосфатурии [22]. С другой стороны, повышение ФРФ-23 может оказывать негативное влияние на уровень $1,25(\mathrm{OH})_{2} \mathrm{D}$ в виде его снижения, в долгосрочной перспективе приводя к снижению МПК [23]. Тем не менее, учитывая доклинические и клинические данные, сегодня пока рано говорить о клинически значимом влиянии повышения уровня ФРФ-23 [24] на фоне приема ИНГТ2. Так, после 12 нед лечения мы не наблюдали значимого повышения уровня ПТГ, однако в некоторых других исследованиях лечение ИНГТ2 приводило к его повышению $[16,17]$. Различия в полученных данных могут быть связаны с разными популяциями пациентов и длительностью наблюдений, что требует продолжения исследований в данной области.

В нашем исследовании мы подтвердили предыдущие наблюдения и показали, что ИНГТ2 оказывают влияние на показатели фосфорно-кальциевого обмена: так, на фоне лечения эмпаглифлозином наблюдались значимое увеличение концентрации ФРФ-23 и тенденция к увеличению концентрации фосфора в сыворотке крови. Концентрации кальция, ПТГ и 25(OH)D остались неизменными на фоне лечения эмпаглифлозином. Наши результаты частично согласуются с данными исследования, проведенного у здоровых добровольцев, в котором канаглифлозин в дозе 300 мг/сут повышал уровни ФРФ-23 и ПТГ [18]. Дополнительно Blau et al. показали, что терапия канаглифлозином ассоциирована со снижением концентрации активной формы витамина D - дигидроксивитамина D $(1,25(\mathrm{OH}), \mathrm{D})$. B нашем исследовании мы не оценивали уровень $1,25(\mathrm{OH})_{2} \mathrm{D}$, однако, судя по данным других исследований, снижение концентрации 1,25(OH) 2 व яляется незначительным и отмечается лишь в течение первых нескольких часов после приема препарата [17, 25], существенно не претерпевая изменений в более поздние сроки. Сохраняются ли эти изменения после длительного лечения ИНГТ-2 и остается ли связь с повышением уровня ФРФ-23, до настоящего времени остается неизвестным. Аналогичным образом, de Jong и соавт. показали, что другой сахароснижающий препарат этой группы, дапаглифлозин, также увеличивает уровни Р, ПТГ и ФРФ-23 в сыворотке крови у пациентов с СД2 и начальными стадиями хронической болезни почек [17].

Результаты проведенного исследования подтвердили тот факт, что пациенты с СД2 в большинстве случаев имеют низкий уровень 25(OH)D в сыворотке крови и нормальные показатели MПК по результатам DXA. Вместе с тем определение показателя ТКИ позволяет диагностировать снижение качества костной ткани у 25,6\% пациентов. Согласно результатам проведенных исследований, ТКИ является независимым предиктором риска переломов [26-29]. Кроме этого, есть подтверждение того, что у больных СД2 показатель ТКИ ниже, чем у лиц без диабета, что позволяет рекомендовать его оценку в клинической практике для расчета риска переломов у пациентов с диабетом [30]. 
Клиническая значимость результатов

Клиническая значимость полученных результатов требует уточнения в ходе дальнейших исследований, поскольку до конца не ясно, насколько длительно сохраняются выявленные изменения и влияют ли они на конечные точки.

\section{Ограничения исследования}

У проведенного исследования есть ряд ограничений, таких как небольшое количество субъектов исследования, небольшая продолжительность наблюдения и отсутствие контрольной группы. Кроме того, в ходе исследования не контролировалось потребление фосфатов с пищей, а также не проводилась оценка концентрации 1,25(OH) 2 D.

\section{Направления дальнейших исследований}

Требуется дальнейшее проведение исследований на больших выборках с контрольной группой как у пациентов с СД2 и сохранной функцией почек, так и у пациентов с СД2 и хронической болезнью почек.

\section{ЗАКЛЮЧЕНИЕ}

По результатам проведенного исследования терапия эмпаглифлозином у больных СД2 с сохранной функцией почек на протяжении 12 нед была ассоциирована с увеличением концентрации ФРФ-23 без значимого влияния на уровни $\mathrm{P}, \mathrm{Ca}, 25(\mathrm{OH}) \mathrm{D}$ и ПТГ. Поскольку вопрос о развитии переломов не является класс-эффектом ИНГТ2, клиническое значение таких изменений требует дальнейшего изучения.

\section{ДОПОЛНИТЕЛЬНАЯ ИНФОРМАЦИЯ}

Источники финансирования. Исследование выполнено за счет средств гранта РНФ № 17-75-30052, Государственного задания №18.

Конфликт интересов. Авторы декларируют отсутствие явных и потенциальных конфликтов интересов, связанных с содержанием настоящей статьи.

Участие авторов. Лебедев Д.А. - анализ полученных данных, написание текста; Тимкина Н.В. - сбор и обработка материалов; Каронова Т.Л. концепция и дизайн исследования, анализ полученных данных, написание текста; Андреева А.Т. - сбор и обработка материалов; Кокина М.А. - сбор и обработка материалов; Григорьева А.Л. - сбор и обработка материалов; Бабенко А.Ю. - концепция и дизайн исследования; Гринева Е.Н. анализ полученных данных, написание текста.

Все авторы одобрили финальную версию статьи перед публикацией, выразили согласие нести ответственность за все аспекты работы, подразумевающую надлежащее изучение и решение вопросов, связанных с точностью или добросовестностью любой части работы.

\section{СПИСОК ЛИТЕРАТУРЫ | REFERENCES}

1. Leslie WD, Rubin MR, Schwartz AV, Kanis JA. Type 2 diabetes and bone. J Bone Miner Res. 2012;27(11):2231-2237. doi: https://doi.org/10.1002/jbmr.1759.

2. Billington $\mathrm{EO}$, Grey $\mathrm{A}$, Bolland MJ. The effect of thiazolidinediones on bone mineral density and bone turnover: systematic review and meta-analysis. Diabetologia. 2015;58(10):2238-2246. doi: https://doi.org/10.1007/s00125-015-3660-2

3. Russo GT, Giandalia A, Romeo EL, et al. Fracture Risk in Type 2 Diabetes: Current Perspectives and Gender Differences. Int J Endocrinol. 2016;2016:1-11. doi: https://doi.org/10.1155/2016/1615735

4. Napoli N, Strotmeyer ES, Ensrud KE, et al. Fracture risk in diabetic elderly men: the MrOS study. Diabetologia. 2014;57(10):2057-2065 doi: https://doi.org/10.1007/s00125-014-3289-6

5. Oei L, Zillikens MC, Dehghan A, et al. High Bone Mineral Density and Fracture Risk in Type 2 Diabetes as Skeletal Complications of Inadequate Glucose Control: The Rotterdam Study. Diabetes Care. 2013;36(6):1619-1628. doi: https://doi.org/10.2337/dc12-1188

6. Ulivieri FM, Silva BC, Sardanelli F, et al. Utility of the trabecular bone score (TBS) in secondary osteoporosis. Endocrine. 2014;47(2):435-448. doi: https://doi.org/10.1007/s12020-014-0280-4

7. Bonaccorsi G, Fila E, Messina C, et al. Comparison of trabecular bone score and hip structural analysis with FRAX ${ }^{\circledR}$ in postmenopausal women with type 2 diabetes mellitus. Aging Clin Exp Res. 2017;29(5):951-957. doi: https://doi.org/10.1007/s40520-016-0634-2

8. Zhukouskaya WV, Ellen-Vainicher C, Gaudio A, et al. The utility of lumbar spine trabecular bone score and femoral neck bone mineral density for identifying asymptomatic vertebral fractures in well-compensated type 2 diabetic patients. Osteoporos Int. 2016;27(1):49-56. doi: https://doi.org/10.1007/s00198-015-3212-0

9. Andreeva A, Burmistrova D, Grigorieva A, et al. Bone mineral density and 10-year probability of fractures in type 2 diabetic patients with different vitamin D status. Endocr Abstr. 2019;63:484. doi: https://doi.org/10.1530/endoabs.63.P484

10. US Food and Drug Administration. Safety alert on canagliflozin, issued on Oct 10, 2015. [cited 29 July 2016]. Available from: http://www.fda.gov/Safety/MedWatch/SafetyInformation/ SafetyAlertsforHumanMedic alProducts/ucm4 61876.htm

11. Monami M, Nardini C, Mannucci E. Efficacy and safety of sodium glucose co-transport-2 inhibitors in type 2 diabetes: a metaanalysis of randomized clinical trials. Diabetes, Obes Metab. 2014;16(5):457-466. doi: https://doi.org/10.1111/dom.12244
12. Wanner C, Inzucchi SE, Lachin JM, et al. Empagliflozin and Progression of Kidney Disease in Type 2 Diabetes. N Engl J Med. 2016;375(4):323-334. doi: https://doi.org/10.1056/NEJMoa1515920

13. Wiviott SD, Raz I, Bonaca MP, et al. Dapagliflozin and Cardiovascular Outcomes in Type 2 Diabetes. N Eng/ J Med. 2019;380(4):347-357. doi: https://doi.org/10.1056/NEJMoa1812389

14. Heerspink HJL, Desai M, Jardine M, et al. Canagliflozin Slows Progression of Renal Function Decline Independently of Glycemic Effects. J Am Soc Nephrol. 2017;28(1):368-375. doi: https://doi.org/10.1681/ASN.2016030278

15. Ruanpeng D, Ungprasert P, Sangtian J, Harindhanavudhi T. Sodium-glucose cotransporter 2 (SGLT2) inhibitors and fracture risk in patients with type 2 diabetes mellitus: A meta-analysis. Diabetes Metab Res Rev. 2017;33(6):e2903. doi: https://doi.org/10.1002/dmrr.2903

16. Alba M, Xie J, Fung A, Desai M. The effects of canagliflozin a sodium glucose co-transporter 2 inhibitor, on mineral metabolism and bone in patients with type 2 diabetes mellitus. Curr Med Res Opin. 2016;32(8):1375-1385. doi: https://doi.org/10.1080/03007995.2016.1174841

17. de Jong MA, Petrykiv SI, Laverman GD, et al. Effects of Dapagliflozin on Circulating Markers of Phosphate Homeostasis. Clin J Am Soc Nephrol. 2019;14(1):66-73. doi: https://doi.org/10.2215/CJN.04530418

18. Blau JE, Bauman V, Conway EM, et al. Canagliflozin triggers the FGF23/1,25-dihydroxyvitamin D/PTH axis in healthy volunteers in a randomized crossover study. JCI Insight. 2018;3(8):1-14. doi: https://doi.org/10.1172/jci.insight.99123.

19. Perkovic V, Jardine MJ, Neal B, et al. Canagliflozin and Renal Outcomes in Type 2 Diabetes and Nephropathy. N Engl J Med. 2019:380(24):2295-2306. doi: https://doi.org/10.1056/NEJMoa1811744

20. Мельниченко Г.А., Белая Ж.Е., Рожинская Л.Я., и др. Федеральные клинические рекомендации по диагностике, лечению и профилактике остеопороза // Проблемы эндокринологии. 2017. — T. 63. — №6. — C. 392-426. [Mel'nichenko GA, Belaya ZE, Rozhinskaya LY, et al. Russian federal clinical guidelines on the diagnostics, treatment, and prevention of osteoporosis. Problems of Endocrinology. 2018;63(6):392-426. (In Russ.)]. doi: https://doi.org/10.14341/probl2017636392-426 
21. Kohler S, Zeller C, Iliev H, Kaspers S. Safety and Tolerability of Empagliflozin in Patients with Type 2 Diabetes: Pooled Analysis of Phase I-III Clinical Trials. Adv Ther. 2017;34(7):1707-1726. doi: https://doi.org/10.1007/s12325-017-0573-0

22. Гребенникова Т.А., Белая Ж.Е., Цориев Т.Т., и др. Эндокринная функция костной ткани // Остеопороз и остеопатuи. - 2015. T. 18. - №1. - C. 28-37. [Grebennikova TA, Belaya ZhE, Tsoriev TT, et al. The endocrine function of the bone tissue. Osteoporosis and Bone Diseases. 2015;18(1):28-37. (In Russ.)].

23. Taylor SI, Blau JE, Rother KI. Possible adverse effects of SGLT2 inhibitors on bone. Lancet Diabetes Endocrinol. 2015;3(1):8-10. doi: https://doi.org/10.1016/S2213-8587(14)70227-X

24. Quarles LD. Skeletal secretion of FGF-23 regulates phosphate and vitamin D metabolism. Nat Rev Endocrinol. 2012;8(5):276-286. doi: https://doi.org/10.1038/nrendo.2011.218

25. Ljunggren Ö, Bolinder J, Johansson L, et al. Dapagliflozin has no effect on markers of bone formation and resorption or bone mineral density in patients with inadequately controlled type 2 diabetes mellitus on metformin. Diabetes, Obes Metab. 2012;14(11):990-999. doi: https://doi.org/10.1111/j.1463-1326.2012.01630.x
26. Hans D, Goertzen AL, Krieg M-A, Leslie WD. Bone microarchitecture assessed by TBS predicts osteoporotic fractures independent of bone density: The manitoba study. J Bone Miner Res. 2011;26(11):2762-2769. doi: https://doi.org/10.1002/jbmr.499

27. McCloskey EV, Odén A, Harvey NC, et al. A Meta-Analysis of Trabecular Bone Score in Fracture Risk Prediction and Its Relationship to FRAX. J Bone Miner Res. 2016;31(5):940-948. doi: https://doi.org/10.1002/jbmr.2734

28. Greendale GA, Huang M, Cauley JA, et al. Premenopausal and early postmenopausal trabecular bone score (TBS) and fracture risk: Study of Women's Health Across the Nation (SWAN). Bone. 2020;140:115543. doi: https://doi.org/10.1016/j.bone.2020.115543

29. Florez H, Hernández-Rodríguez J, Muxi A, et al. Trabecular bone score improves fracture risk assessment in glucocorticoidinduced osteoporosis. Rheumatology. 2020;59(7):1574-1580. doi: https://doi.org/10.1093/rheumatology/kez464

30. Holloway KL, De Abreu LLF, Hans D, et al. Trabecular Bone Score in Men and Women with Impaired Fasting Glucose and Diabetes. Calcif Tissue Int. 2018;102(1):32-40. doi: https://doi.org/10.1007/s00223-017-0330-z

\section{ИНФОРМАЦИЯ ОБ АВТОРАХ [AUTHORS INFO]}

* Лебедев Денис Андреевич [Denis A. Lebedev]; адрес: 194156, Россия, Санкт-Петербург, пр. Пархоменко, д. 15 [address: 15 Parkhomenko street, Russia, St. Petersburg, 194156]; ORCID: https://orcid.org/0000-0003-1808-1331; eLibrary SPIN: 3588-2999; e-mail: doctorlebedev11@gmail.com

Тимкина Наталья Владимировна [Natalia V. Timkina]; ORCID: https://orcid.org/0000-0001-9836-5427; eLibrary SPIN: 6259-7745; e-mail: n.timkina2014@yandex.ru

Каронова Татьяна Леонидовна, д.м.н., професcop [Tatiana L. Karonova, MD, PhD, professor]; ORCID: https://orcid.org/0000-0002-1547-0123; eLibrary SPIN: 3337-4071; e-mail: karonova@mail.ru

Андреева Алена Тимуровна [Alena T. Andreeva]; ORCID: https://orcid.org/0000-0002-4878-6909; eLibrary SPIN: 6051-7214; e-mail: arabicaa@gmail.com

Кокина Мария Александровна [Maria A. Kokina]; ORCID: https://orcid.org/0000-0002-2882-9406; e-mail: mapillika@yandex.ru

Григорьева Александра Леонидовна [Alexandra L. Grigorieva]; ORCID: https://orcid.org/0000-0003-0888-8991; e-mail: alexagri@mail.ru

Бабенко Алина Юрьевна, Д.М.н., профессор [Alina Yu. Babenko, MD, PhD, professor]; ORCID: https://orcid.org/0000-0002-0559-697X; eLibrary SPIN: 9388-1077; e-mail: alina_babenko@mail.ru

Гринева Елена Николаевна, д.м.н., професcop [Elena N. Grineva, MD, PhD, professor];

ORCID: https://orcid.org/0000-0002-0559-697X; eLibrary SPIN: 2703-0841; e-mail: grineva_e@mail.ru

\section{ИНФОРМАЦИЯ}

Рукопись получена: 19.03.2021. Одобрена к публикации: 18.06.2021.

\section{ЦИТИРОВАТЬ:}

Лебедев Д.А., Тимкина Н.В., Каронова Т.Л., Андреева А.Т., Кокина М.А., Григорьева А.Л., Бабенко А.Ю., Гринева Е.Н. Влияние эмпаглифлозина на фосфорно-кальциевый метаболизм у больных сахарным диабетом 2 типа с сохранной функцией почек // Остеопороз и остеоnатии. — 2021. — T. 24. — №1. — C. 4-9. doi: https://doi.org/10.14341/osteo12718

\section{TO CITE THIS ARTICLE:}

Lebedev DA, Timkina NV, Karonova TL, Andreeva AT, Kokina MA, Grigorieva AL, Babenko AYu, Grineva EN. Effect of empagliflozin on phosphorus and calcium metabolism in patients with type 2 diabetes mellitus with preserved kidney function. Osteoporosis and bone diseases. 2021;24(1):4-9. doi: https://doi.org/10.14341/osteo12718 